\title{
OPEN Microstructural degeneration and cerebrovascular risk burden underlying executive dysfunction after stroke
}

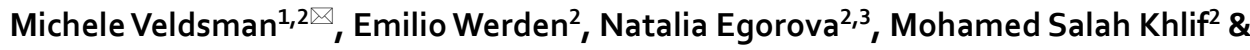 \\ Amy Brodtmann ${ }^{2,4,5}$
}

Executive dysfunction affects $40 \%$ of stroke patients, but is poorly predicted by characteristics of the stroke itself. Stroke typically occurs on a background of cerebrovascular burden, which impacts cognition and brain network structural integrity. We used structural equation modelling to investigate whether measures of white matter microstructural integrity (fractional anisotropy and mean diffusivity) and cerebrovascular risk factors better explain executive dysfunction than markers of stroke severity. 126 stroke patients (mean age 68.4 years) were scanned three months post-stroke and compared to 40 age- and sex-matched control participants on neuropsychological measures of executive function. Executive function was below what would be expected for age and education level in stroke patients as measured by the organizational components of the Rey Complex Figure Test, $\mathrm{F}(3,155)=17, \mathrm{R}^{2}=0.25, p<0.001$ (group significant predictor at $p<0.001$ ) and the Trail-Making Test $(B), F(3,157)=3.70, R^{2}=0.07, p<0.01$ (group significant predictor at $p<0.001$ ). A multivariate structural equation model illustrated the complex relationship between executive function, white matter integrity, stroke characteristics and cerebrovascular risk (root mean square error of approximation $=0.02$ ). Pearson's correlations confirmed a stronger relationship between executive dysfunction and white matter integrity $(r=-0.74, p<0.001)$, than executive dysfunction and stroke severity $(r=0.22, p<0.01)$. The relationship between executive function and white matter integrity is mediated by cerebrovascular burden. White matter microstructural degeneration of the superior longitudinal fasciculus in the executive control network better explains executive dysfunction than markers of stroke severity. Executive dysfunction and incident stroke can be both considered manifestations of cerebrovascular risk factors.

Cognitive impairment after stroke is associated with impaired activities of daily living ${ }^{1}$ and increased mortality ${ }^{2}$. Executive dysfunction is a major component of vascular and post-stroke cognitive impairment, occurring in around $40 \%$ of patients after ischemic stroke, and predicting quality of life, even when controlling for age, depression and stroke severity ${ }^{1,2}$. Stroke location alone has not been systematically associated with the development of post-stroke executive impairment, likely because stroke imaging cohorts have been too small and variable to detect consistent relationships between different cognitive functions and stroke locations. In addition, stroke has widespread effects across the brain ${ }^{3-6}$. There is some evidence that strokes affecting anterior circulation ${ }^{1}$ or frontal circuits ${ }^{7}$ are more likely to result in executive impairment, but executive impairment has been reported in cohorts of patients with a mix of stroke locations and etiologies ${ }^{8-11}$. It has not proven possible to predict executive dysfunction based simply on the clinical characteristics of a patient's stroke.

Stroke occurs on a background of aging and cerebrovascular risk (CVR) factors, which predominantly impact executive function ${ }^{12,13}$. Ten modifiable risk factors, predominantly affecting cerebrovascular are associated with $90 \%$ of the risk of stroke ${ }^{14}$. Modifiable risk factors that contribute to cerebrovascular burden, such as smoking and hypertension, impact brain network integrity ${ }^{15,16}$. The best known macroscale markers of cerebrovascular

\footnotetext{
${ }^{1}$ Department of Experimental Psychology, University of Oxford, New Radcliffe House, Radcliffe Observatory Quarter, Oxford OX2 6GG, UK. ${ }^{2}$ The Florey Institute of Neuroscience and Mental Health, University of Melbourne, Melbourne, Australia. ${ }^{3}$ Melbourne School of Psychological Sciences, University of Melbourne, Melbourne, VIC, Australia. ${ }^{4}$ Austin Health, Heidelberg, Melbourne, VIC, Australia. ${ }^{5}$ Eastern Clinical Research Unit, Box Hill Hospital, Melbourne, VIC, Australia. ${ }^{\square}$ email: michele.veldsman@psy.ox.ac.uk
} 
burden are white matter hyperintensities of presumed vascular origin (WMHs) ${ }^{17}$. Yet, the relationship between WMHs and executive function is far from clear ${ }^{18}$.

Although many definitions of executive function exists, most investigators agree higher order cognitive components of executive function include planning, organizing, and maintaining strategies for controlling and executing behavior, allocating attention, and inhibiting distracting information ${ }^{1}$. Executive function is closely associated with the integrity of the prefrontal $\operatorname{cortex}^{19}$, but damage to white matter connections to other brain regions can also impair function ${ }^{20}$. Structural and functional brain network integrity is therefore critical to maintaining cognitive function ${ }^{21}$. Brain networks show a high degree of functional specificity ${ }^{22}$. The canonical frontoparietal brain networks subserve higher cognitive functions related to attention and executive function ${ }^{23,24}$.

The superior longitudinal fasciculus (SLF) is the principal white matter tract connecting the frontal and parietal nodes of the frontoparietal network ${ }^{23}$. Within the SLF, dorsal, middle and ventral branches connect superior, intraparietal and inferior parietal and frontal regions to superior, middle and inferior frontal regions, respectively ${ }^{23}$ : an architecture preserved from the monkey brain. Collectively, these divisions facilitate communication between regions of the frontoparietal network underlying broad executive control functions ${ }^{23,24}$.

Vascular risk factors may also impact on the normal appearing white matter of brain networks ${ }^{25}$. Microstructural markers measured from magnetic resonance diffusion weighted images can act as surrogate markers of brain network integrity. These markers show a decline in ageing that is accelerated in the presence of CVR factors. For example, high blood pressure is associated with disrupted white matter microstructural integrity, even when controlling for age and the presence of white matter hyperintensities ${ }^{26}$. Decreased executive function was associated with altered functional connectivity of the frontoparietal network in 1007 elders, with reduced white matter integrity specific to the SLF in hypertensive compared to normotensive individuals ${ }^{27}$. Likewise, in cerebral small vessel disease and lacunar stroke, white matter microstructure better predicts executive function than macroscale markers of cerebrovascular burden such as white matter hyperintensities ${ }^{28}$.

Despite relatively focal damage caused by stroke, remote effects occur across distributed brain networks ${ }^{3}$. The predictors of post-stroke executive dysfunction remain unclear, despite executive deficits commonly impacting post-stroke quality of life. It is likely that the various impacts of vascular brain burden are better explored using a multifaceted model to explain executive function after stroke, taking into account ageing, background cerebrovascular burden, measures of global brain health and brain network integrity. We used structural equation modelling to examine the multivariate relationships underlying executive dysfunction after stroke. We hypothesized that executive dysfunction would be well predicted by the integrity of the SLF, not characteristics of the stroke itself. We also predicted that background cerebrovascular risk burden would play an important role in mediating brain health and executive function.

\section{Methods}

We analyzed data from 135 stroke patients and 40 healthy controls from the Cognition and Neocortical Volume After Stroke study (CANVAS) ${ }^{29}$. All participants gave written, informed consent in line with the Declaration of Helsinki and the study was approved by human research committees of Austin Health, Eastern Health and Melbourne Health. All methods were carried out in accordance with the relevant guidelines within the Declaration of Helsinki. Patients with hemorrhagic stroke, significant comorbidities, pre-existing dementia, or without a proven clinical ischemic stroke on imaging were excluded from participation. Patients underwent MRI scanning and comprehensive neuropsychological testing at repeated intervals over three years. Healthy, age matched controls underwent the same sessions at equivalent intervals. The data from three months post-stroke were analysed here. We specifically chose the three-month time-point to analyse because it is the commonest time-point for stroke outcome reportage in stroke intervention studies. Our baseline time-point for this study included participants imaged while dynamic changes were occurring, such as oedema resolution. We believe that the three-month time-point is an ideal snapshot of the effects of stroke on executive function, as it captures both the effect of the stroke and concomitant vascular brain burden. Participants were interviewed to record vascular risk factors and medications, with information cross-referenced against hospital records. As part of a battery of neuropsychological testing, participants completed the Trail-Making Test (B) ${ }^{30}$, the Clock Drawing Task $^{31}$ and the Rey Complex Figure Test ${ }^{32}$. These tests were included in the executive function domain score. The neuropsychological measures were chosen for each domain in the CANVAS study by an expert panel made up of two cognitive neurologists, two research neuropsychologists and a registered clinical neuropsychologist (as outlined in the published protocol ${ }^{29}$.

Cerebrovascular risk score. A cerebrovascular risk score was calculated for each participant based on the sum of known risk factors. Individual risk factors were thresholded according to published guidelines for the management of cerebrovascular risk ${ }^{33}$. A body mass index greater than $30 \mathrm{~kg} / \mathrm{m}^{2}$, diagnosed or medicated hypertension, cholesterol, Type II Diabetes, ischemic heart disease, or atrial fibrillation contributed to the score. Positive smoking status and drinking greater than 14 units per week also contributed to the score. We used a bespoke score created with the available vascular risk data collected as part of the CANVAS ${ }^{29}$ study.

Image acquisition. Magnetic resonance images were acquired on a Siemens $3 \mathrm{~T}$ scanner with a 12-channel head coil. MRI included a fluid attenuated inversion recovery image (FLAIR), diffusion weighted imaging (DWI) and high-resolution structural magnetization prepared rapid acquisition gradient echo (MPRAGE) image, as part of a longer protocol ${ }^{29}$. A 160 sagittal slice MPRAGE had $1 \mathrm{~mm}$ isotropic voxels, $1900 \mathrm{~ms}$ repetition time (TR), $2.55 \mathrm{~ms}$ echo time (TE), $9^{\circ}$ flip angle and $256 \times 256$ acquisition matrix. The 3 -D SPACE FLAIR had $160,1 \mathrm{~mm}$ thick sagittal slices, a TR of $6000 \mathrm{~ms}$, a TE of $380 \mathrm{~ms}$, a $120^{\circ}$ flip angle and a $256 \times 254$ acquisition matrix. Sixty volumes of single shot spin echo EPI with a TR $8.4 \mathrm{~s}$, TE $110 \mathrm{~ms}$ were collected, with 60 diffusion 
sensitization directions, $b=3000 \mathrm{~s} / \mathrm{mm}^{2}$ and $2.5 \mathrm{~mm}$ isotropic voxels. Additional $b=0$ reverse phase-encoded images were acquired to aid in geometric distortion correction.

Imaging analysis. Stroke infarcts were verified by a stroke neurologist $(\mathrm{AB})$ and manually delineated by an imaging researcher expert in lesion tracing (MSK). The total volume of lesion was estimated from the binary manual mask. White matter hyperintensities (WMH) were segmented automatically using the Wisconsin WMH Segmentation Toolbox (W2MHS) ${ }^{34}$. Classification of WMHs was based on a supervised Random Forest-based regression. Both 3D FLAIR and MPRAGE images were used in the segmentation process. Total WMH load was calculated for each participant.

Diffusion images were preprocessed in the Diffusion Toolbox (FDT) in the fMRIB Software Library (FSL) using a standard pipeline. The "topup" function in FDT estimated susceptibility induced distortions and was input into the "eddy" function for correction of movement and gradient coil distortions. Diffusion tensors were fit using the inbuilt DTIFIT function. Fractional anisotropy (FA) and mean diffusivity (MD) values were extracted from the superior longitudinal fasciculus (SLF) as defined by the John Hopkins University tractography atlas ${ }^{35}$. Nine stroke participants were excluded due to imaging artefacts in their scans as a result of movement or problems with registration of images that could not be manually fixed.

Statistical analysis. Executive function was compared in the stroke and healthy control group using Welch's independent t-tests, Holm-Bonferroni corrected for multiple comparisons, in the JASP statistical package. Performance in each test was normalized against age-matched, published norms. Multiple regression was used to further explore this relationship correcting for age and years of education between groups.

Structural equation modelling (SEM) was carried out in the R Lavaan Package ${ }^{36}$. Only stroke patients were included in the SEM as central to the model was the impact of stroke characteristics. Structural equation modelling is a multivariate technique that enables estimation of the relationship between multiple variables including latent variables (constructs that cannot be directly measured) and observed variables (that can be measured directly). SEM typically combines a measurement model (how observed variables relate to latent variables) and a structural model (how latent variables relate to each other). Our model included a latent variable representing frontoparietal white matter integrity, made up of mean FA and MD of the superior longitudinal fasciculus. A second latent variable represented executive impairment with indicators based on performance in the three executive cognitive tasks. A third latent variable indexed stroke severity and included the volume of the lesion, the National Institute of Health Stroke Scale (NIHSS) score and the modified Rankin Scale (mRS) three months post-stroke. Confirmatory factor analysis predicted the contribution of the latent variables to their indicator variables. Observed variables included age, WMH load and cerebrovascular risk score. Missing data were estimated using full information maximum likelihood (FIML). Ten percent of the data points were missing, as there was no consistency in which variables or individuals were missing data, the missingness was treated as random.

Nested model testing was used to confirm the model was a good fit for the data and assess the contribution of the frontoparietal white matter integrity latent variable. Multiple model fit indices were used to determine if the model was a good fit for the data.

Latent variables were extracted using a built-in function in the Lavaan package and correlated with observed variables using Pearson's correlations and a significance level set to $p<0.05$. Mediation analysis was used to further clarify the relationship between executive dysfunction, frontoparietal white matter integrity, stroke severity and cerebrovascular risk. Simple mediation analysis was conducted in Lavaan with a maximum likelihood estimator. Assumptions of mediation analysis are the same as those for the general linear model, including normal distribution of the variables and homogeneity of error variances which were met in the current analysis. Skewness for the executive dysfunction, frontoparietal white matter integrity, stroke severity and cerebrovascular risk variables were $0.48,0.03,0.69$ and 0.36 indicating the data are fairly symmetrical ( 0 indicating the perfect symmetry of a normal distribution).

\section{Results}

Stroke patients and controls were well matched in terms of age and ratio of male to female (Table 1). As can be seen in the stroke lesion overlap map (Fig. 1), the sites of stroke damage varied across patients with more right $(n=82)$ than left hemisphere $(n=50$, three bilateral) strokes. Using the accepted Oxfordshire Community Stroke Project ${ }^{37}$ classification, $1 \%$ (2) of the strokes were classified as total anterior circulation infarcts (TACI), 52\% (70) were partial anterior circulation infarcts (PACI), $14 \%$ (19) were lacunar infarcts (LACI), and 33\% (44) were posterior circulation infarcts (POCI).

Welch's independent t-tests confirmed that stroke patients were impaired on the Rey Complex Figure Test, $\mathrm{t}(78)=5.75, p<0.001$, and the Trail-Making Test (B), $\mathrm{t}(125)=4.23, p<0.001$ (Fig. 2). Patients and healthy controls performed to a similar level on the Clock Drawing Task $\mathrm{t}(82)=1.81, p=0.07$ (Fig. 2). Given a significant difference in the median years of education between stroke patients and healthy controls (Table 1), multiple regressions were run to control for age and education level. Even after controlling for age and education level, stroke was a significant predictor of performance in the Rey Complex Figure Test, $\mathrm{F}(3,155)=17, \mathrm{R}^{2}=0.25, p<0.001$ (group significant predictor at $p<0.001$ ); and the Trail-Making Test $(\mathrm{B}), \mathrm{F}(3,157)=3.70, \mathrm{R}^{2}=0.07, p<0.01$ (group significant predictor at $p<0.001$ ) but not the Clock Drawing Task, $\mathrm{F}(3,160)=2.01, \mathrm{R}^{2}=0.04, p=0.12$ (group non-significant predictor at $p=0.13$ ).

Multiple model fit indices confirmed that our full structural equation model (Fig. 3a) was a very good fit for the data. Comparative Fit index was 0.99 for the full model (Fig. 3a) and 0.80 for the constrained model (Fig. 3b). The Tucker-Lewis Index, which penalizes overly complex models, was 0.99 for model A and 0.71 for model B. For both these fit indices, values greater than 0.9 indicate good model $\mathrm{fit}^{38}$. The absolute fit index, root mean 


\begin{tabular}{|c|c|c|c|}
\hline \multirow[b]{2}{*}{ Demographics } & \multicolumn{2}{|l|}{ Statistics } & \multirow[b]{2}{*}{ Group difference } \\
\hline & Stroke patients & Healthy controls & \\
\hline Number (female) & $135(41)$ & $40(15)$ & Chi Square $\mathrm{X}^{2}=0.72, p=0.40$ \\
\hline Mean age (SD) & $68(11.8)$ & $69(6.6)$ & Welch's $\mathrm{t}(120)=0.26, p=0.80$ \\
\hline Right handed:Left handed & $125: 10$ & $36: 4$ & Chi Square $\mathrm{X}^{2}=0.28, p=0.60$ \\
\hline Median years of education (IQR 1,3 ) & $12(10,15)$ & $17(11,18)$ & Mann-Whitney $w=3689, p<0.001$ \\
\hline Mean MoCA (SD) & $23(4.1)$ & $26(2.2)$ & Welch's $\mathrm{t}(119)=4.99, p<0.001$ \\
\hline Median NIHSS (IQR 1,3) & $0(0,1)$ & - & $\begin{array}{l}\text { Change in NIHSS baseline-3 months, Wilcoxon Signed Rank test } \\
\mathrm{z}=4437, p<0.001\end{array}$ \\
\hline Median mRS (IQR 1,3) & $1(1,2)$ & - & - \\
\hline Median CVR Score (IQR 1,3) & $2(2,4)$ & $1(1,2)$ & Mann-Whitney $\mathrm{w}=-1712, p<0.001$ \\
\hline Body Mass Index $\left(\%>30 \mathrm{~kg} / \mathrm{m}^{2} /^{2}\right)$ & 30 & 18 & - \\
\hline Percent diagnosed/medicated hypertension & 63 & 43 & - \\
\hline Percent diagnosed/medicated hypercholesteremia & 46 & 35 & - \\
\hline Percent with type II diabetes & 34 & 10 & - \\
\hline Percent with ischaemic heart disease & 25 & 0.03 & - \\
\hline Percent diagnosed atrial fibrillation & 24 & 0.03 & - \\
\hline Percentage of smokers & 47 & 33 & - \\
\hline Percent drinking $>14$ units alcohol per week & 10 & 18 & - \\
\hline Mean stroke lesion volume in $\mathrm{mm}^{3}(\mathrm{SD})$ & $7979(13,810)$ & - & - \\
\hline Mean white matter hyperintensity volume $\mathrm{mm}^{3}$ (SD) & $19(8.3)$ & $10(2.7)$ & Welch's $\mathrm{t}(145)=-9.78, p<0.0001$ \\
\hline Mean fractional anisotropy in superior longitudinal fasciculus (SD) & $0.43(0.04)$ & $0.46(0.01)$ & Welch's $\mathrm{t}(144)=6.52, p<0.0001$ \\
\hline $\begin{array}{l}\text { Mean mean diffusivity in the superior longitudinal fasciculus (cube } \\
\text { transformed)(SD) }\end{array}$ & $0.08(0.002)$ & $0.08(0.00001)$ & Welch's $\mathrm{t}(134)=-5.52, p<0.0001$ \\
\hline
\end{tabular}

Table 1. Patient and control demographics at the 3 month time-point. Group demographics at three month timepoint. IQR, interquartile range; SD, standard deviation; MoCA, Montreal Cognitive Assessment; CVR, cerebrovascular risk score; NIHSS, National Institute of Health Stroke Scale; mRS, Modified Rankin Scale.

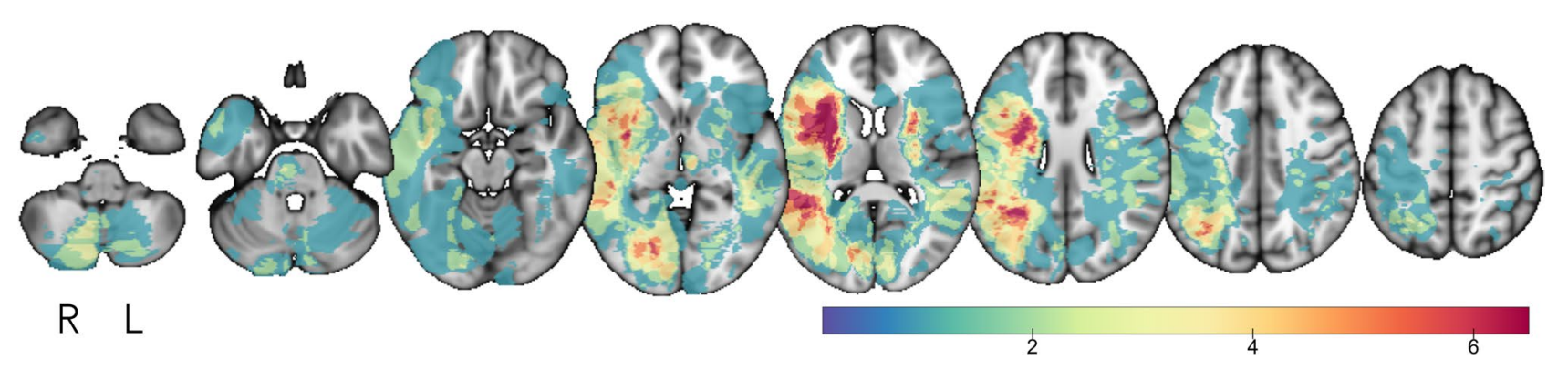

Figure 1. Lesion overlap map. Lesion overlap map displayed on Montreal Neurological Institute template. Colorbar indicates maximum number of overlapping lesions.

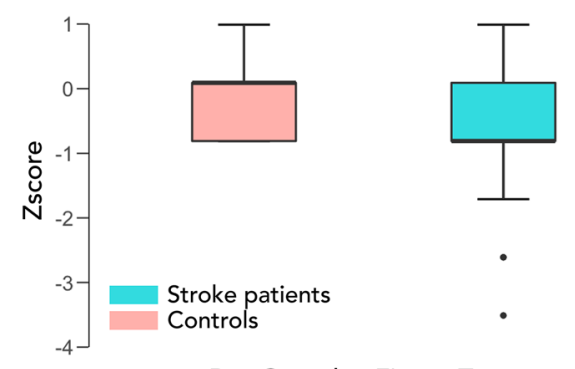

Rey Complex Figure Test

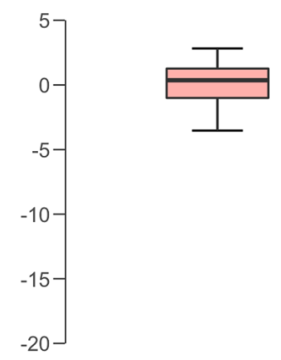

Trail Making Test B
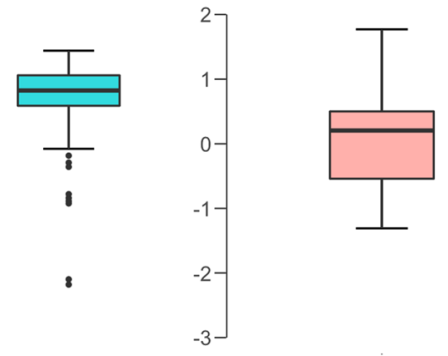

Clock Drawing Task

Figure 2. Executive dysfunction in stroke patients compared to age-matched controls. Boxplots displaying performance (z-score) in stroke patients compared to age-matched controls in three tests of executive function, the Rey Complex Figure task (left panel), Trail Making Test B (middle panel), Clock Drawing task (right panel). 
a)

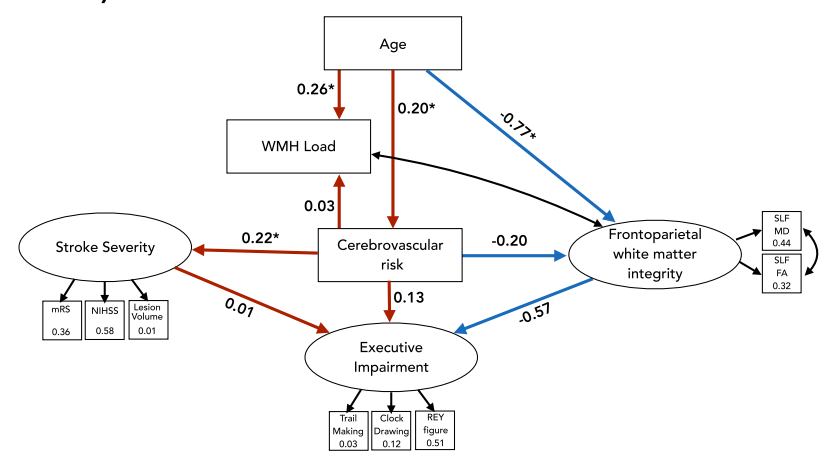

b)

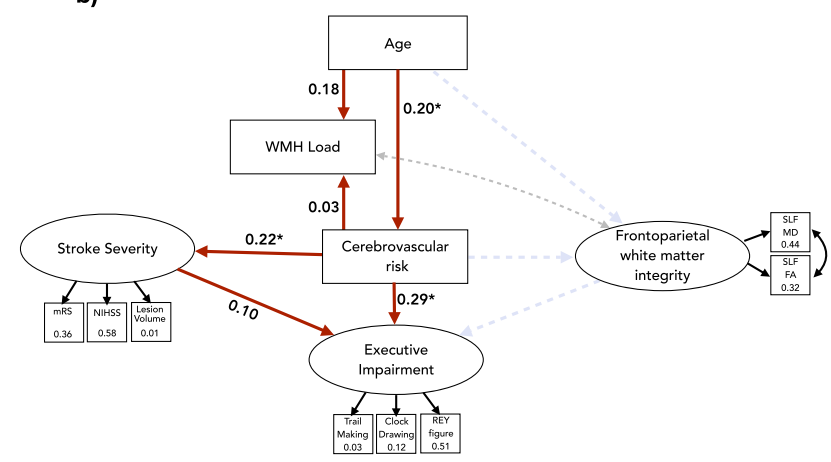

Figure 3. Structural equation path models. Values in square indicators are $\mathrm{R}^{2}$. Values on paths are standardized beta estimates. (a) Full path model. (b) Model constrains the contribution of frontoparietal white matter integrity of the superior longitudinal fasciculus for nested model testing. Red paths show positive directional relationships, blue paths show negative relationships. Black arrows indicate covariances. ${ }^{*}$ paths significant to $p<0.05$.
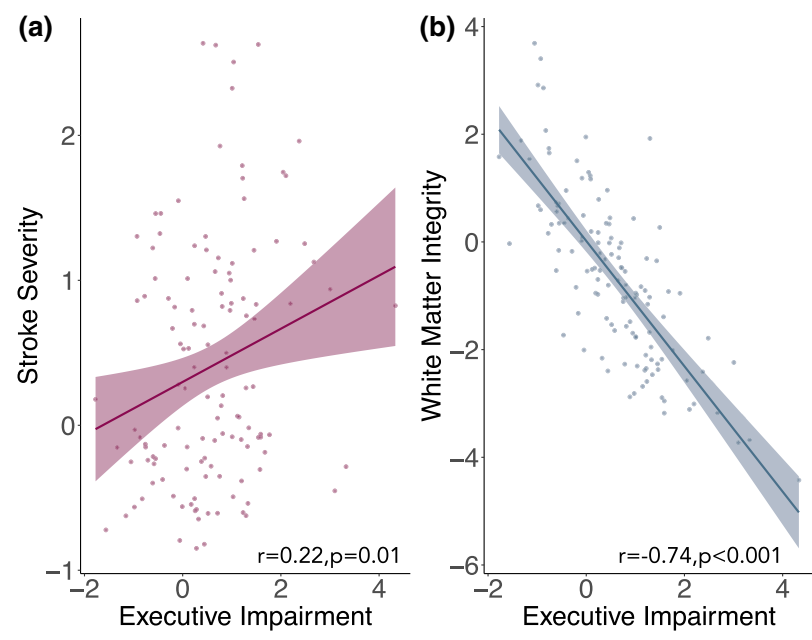

Figure 4. Scatterplots showing latent variable relationships with executive impairment. Correlation between executive impairment and (a) a latent variable representing stroke severity and (b) a latent variable representing white matter integrity of the superior longitudinal fasciculus.

square error of approximation (RMSEA), was 0.02 for model a and 0.10 for model b. As a guideline, RMSEA less than 0.05 is considered a very good fit for the dat ${ }^{38}$. Analysis of variance in Lavaan was used to compare nested model fits and confirmed the full model (model a) was superior to model b, $p<0.0001$.

There was a significant Pearson's correlation between the latent variable representing executive function and stroke severity $\mathrm{r}=0.22, p<0.01$, and executive function and white matter integrity $\mathrm{r}=-0.74, p<0.001$ but not between executive function and cerebrovascular burden, $\mathrm{r}=0.15, p=0.09$. Pearson and Filon's $\mathrm{z}$ test to compare the magnitude of overlapping correlations confirmed that the magnitude of the correlation between executive function and frontoparietal white matter integrity was significantly greater than the magnitude of the correlation between executive function and stroke severity, $z(123)=-9.68, p<0.001$ (Fig. 4). Both measures of white matter integrity used in the latent variable (fractional anisotropy and mean diffusivity); median cerebrovascular risk score and white matter hyperintensity load were all significantly different in stroke patients compared to age-matched controls (Table 1). This confirms reduced white matter integrity of the SLF, increased white matter hyperintensity and higher cerebrovascular risk burden in stroke patients compared to age-matched controls.

Mediation analysis was used to assess the contribution of cerebrovascular risk (Fig. 5a) and stroke severity (Fig. 5b) to the relationship between frontoparietal white matter integrity and executive impairment. Both models were a very good fit for the data (CFI and TLI $=1$, RMSEA $<0.001$ for both models). Confirming the results of the SEM, executive impairment was associated with frontoparietal white matter integrity. Both cerebrovascular risk and stroke severity predicted frontoparietal white matter integrity (standardized beta estimates -0.20 and -0.45 , respectively, $p<0.001$ ). In both models, when the relationship between frontoparietal white matter integrity and executive function is mediated by either CVR or stroke severity the relationship became non-significant (standardized beta estimates -0.004 and -0.02 respectively) indicating complete mediation (Fig. 5). Cerebrovascular 


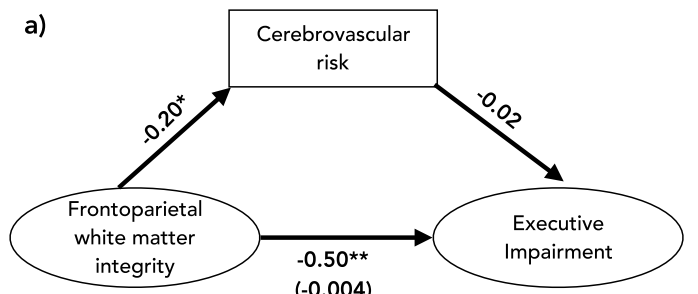

$(-0.004)$

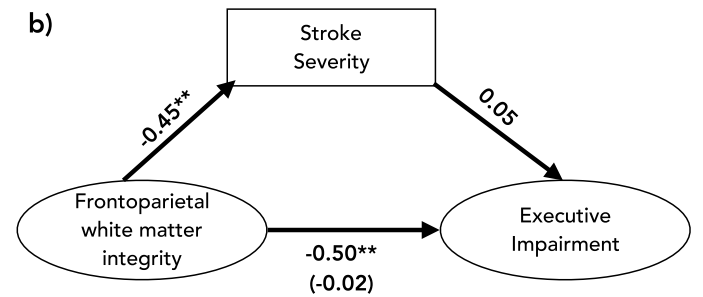

$(-0.02)$

Figure 5. Mediation analysis. Mediation analyses of the relationship between executive impairment and frontoparietal white matter integrity mediated by (a) cerebrovascular risk (CVR) and (b) stroke severity. ${ }^{*}$ path significant to $p<0.05,{ }^{* *} p<0.001$. Value in brackets indicates the standardized beta estimate of the indirect path.

risk (CVR) reduced the relationship between frontoparietal white matter integrity and executive impairment to a greater extent than stroke severity suggesting it has a stronger mediating effect.

\section{Discussion}

We demonstrate executive function below what would be expected for age and education level in a cohort of ischemic stroke patients three months after stroke. This is important because executive dysfunction has been shown to be a significant predictor of quality of life after stroke. A structural equation model was used to assess the complex, multivariate relationship between executive dysfunction and stroke characteristics, cerebrovascular burden and frontoparietal white matter integrity. The location or severity of a stroke on its own cannot predict whether a patient will experience executive dysfunction ${ }^{7}$. Importantly, characteristics of the stroke itself, including measures of functional outcome (mRS), stroke severity (NIHSS) and lesion volume were very poor predictors of executive dysfunction. Nested model-testing confirmed the importance of the integrity of the SLF for predicting executive dysfunction, with significantly worse model fit when SLF integrity was removed from the model. Controversy remains regarding whether conventional clinical stroke markers, such as stroke location, stroke lesion volume and clinical scales such as the National Institute for Health Stroke Scale, are major determinants of cognitive dysfunction after stroke. We believe that our findings confirm that indeed these conventional markers are not the most sensitive, as we found that executive dysfunction was better explained by underlying cerebrovascular burden and white matter integrity. Importantly, we show white matter integrity and cerebrovascular burden is sensitive to executive dysfunction, supporting our hypotheses.

We further interrogated the relationship between executive dysfunction, SLF white matter integrity, cerebrovascular burden and stroke severity, and found the strongest correlation between frontoparietal white matter integrity and executive dysfunction. Mediation analysis revealed that both CVR factors and stroke severity were significant predictors of frontoparietal white matter integrity, confirming the reduced microstructural integrity seen in stroke patients compared to controls. Mediation analysis also confirmed that the significant relationship between executive function and frontoparietal SLF integrity was mediated by cerebrovascular risk and stroke severity. The relationship was no longer significant when controlling for either CVR or stroke severity, with CVR having a stronger mediating effect than stroke severity. This suggests CVR significantly contributes to age-related white matter microstructural degeneration, which in turn impacts on executive dysfunction. Cerebrovascular risk factors are potentially modifiable. Our findings are clinically relevant because control of risk factors may reduce degeneration of frontoparietal white matter integrity, which is already burdened by age-related changes. This implies that risk factor management may reduce the burden of executive dysfunction after stroke.

White matter hyperintensities are the most well-known macroscale marker of cerebrovascular burden, but the relationship between WMH and executive function is not clear, and may reflect more global effects on $\operatorname{cognition}^{39}$. What is less well appreciated, in relation to executive dysfunction after stroke, is the integrity of 'normal appearing white matter' in domain specific cognitive networks. Our model reveals the critical role of microstructural integrity within the SLF which anchors a brain network responsible for executive processing, the frontoparietal network ${ }^{24}$.

The SLF is a major tract in the frontoparietal control network ${ }^{23,40}$ which is specifically vulnerable to the effects of cerebrovascular burden. Ischemic stroke is a clinical manifestation of cumulative vascular risk, but WMHs and brain atrophy from both grey and white matter degeneration occur in the background, with resultant executive dysfunction commonly observed in stroke patients. Our model shows that it may not be the stroke itself, or the stroke alone, that is responsible for executive dysfunction, but rather a combination of degeneration of a major white matter tract underlying a domain-specific distributed brain network and cerebrovascular burden that likely precipitated the stroke event.

It is important to consider the work in the context of some limitations. We only explored the white matter integrity of the superior longitudinal fasciculus, so we cannot say that degeneration is specific to this tract, and may reflect more global white matter degeneration. We used nested model testing to try to establish the importance of frontoparietal white matter integrity and found a model that included it was indeed a better fit (even when being penalized for increasing model complexity). The SEM was limited to stroke patients, because we were interested in estimating the relationship between stroke severity and executive dysfunction. This made it difficult to control for age-related changes, as age is frequently the biggest predictor of cognitive effects. However, our stroke and control group were age matched, and we showed that executive function was impaired in our stroke group, even when controlling for age and education. We further showed that cerebrovascular risk and white 
matter hyperintensity burden is increased in stroke patients and white matter integrity of the SLF is reduced in stroke patients compared to age-matched controls. Finally, because stroke characteristics were estimated using a latent variable for the structural equation modelling, we were unable to include a categorical variable representing stroke location. There is some evidence that patients with strokes affecting fronto-subcortical circuits are more likely to develop executive impairment ${ }^{7}$.

\section{Conclusions}

Executive impairment is common after ischemic stroke and is evident even in a relatively mild stroke cohort. Integrity of normal appearing white matter within a large-scale network specific to executive control, predicts executive dysfunction in stroke patients at three months post-stroke. Control of cerebrovascular risk factors may help to mitigate age-related white matter degeneration.

\section{Data availability}

The data that support the findings of this study are available on reasonable request from the corresponding author. The data are not publicly available as CANVAS is a prospective, "live" study, with expected completion of data acquisition in mid-2020 for the 5-year scanning timepoint. All requests for raw and analysed data will be reviewed by the CANVAS investigators to determine whether the request is subject to any intellectual property or confidentiality obligations.

Received: 8 June 2020; Accepted: 7 October 2020

Published online: 21 October 2020

\section{References}

1. Pohjasvaara, T. et al. Post-stroke depression, executive dysfunction and functional outcome. Eur. J. Neurol. 9, 269-275 (2002).

2. Oksala, N. K. J. et al. Cognitive impairment predicts poststroke death in long-term follow-up. J. Neurol. Neurosurg. Psychiatry 80, 1230-1235 (2009).

3. Veldsman, M. \& Brodtmann, A. Disconnectomics: Stroke-related disconnection and dysfunction in distributed brain networks. Int. J. Stroke 14, 6-8 (2019).

4. Fox, M. D. Mapping symptoms to brain networks with the human connectome. N. Engl. J. Med. 379, 2237-2245 (2018).

5. Amy, B. et al. Dynamic regional brain atrophy rates in the first year after ischemic stroke. Stroke 51, e183-e192 (2020).

6. Veldsman, M. et al. Degeneration of structural brain networks is associated with cognitive decline after ischaemic stroke. (2020) https ://doi.org/10.21203/rs.3.rs-36274/v1.

7. Vataja, R. et al. MRI correlates of executive dysfunction in patients with ischaemic stroke. Eur. J. Neurol. 10, 625-631 (2003).

8. Park, Y. H. et al. Executive function as a strong predictor of recovery from disability in patients with acute stroke: a preliminary study. J. Stroke Cerebrovasc. Dis. 24, 554-561 (2015).

9. Sun, J.-H., Tan, L. \& Yu, J.-T. Post-stroke cognitive impairment: epidemiology, mechanisms and management. Ann. Transl. Med. 2, (2014).

10. Roussel, M. et al. The behavioral and cognitive executive disorders of stroke: the GREFEX study. PLoS ONE 11, e0147602 (2016).

11. Srikanth-Velandai, K. et al. Increased risk of cognitive impairment 3 months after mild to moderate first-ever stroke. Stroke 34, $1136-1143$ (2003).

12. Impaired cognitive function and compliance with antihypertensive drugs in elderly: The Rotterdam Study-Salas-2001—Clinical Pharmacology \& Therapeutics-Wiley Online Library. https://doi.org/10.1016/S0009-9236(01)14469-3.

13. Willcox, B. J. et al. Midlife risk factors and healthy survival in men. JAMA 296, 2343-2350 (2006).

14. O'Donnell, M. J. et al. Global and regional effects of potentially modifiable risk factors associated with acute stroke in 32 countries (INTERSTROKE): a case-control study. The Lancet 388, 761-775 (2016).

15. Friedman, J. I. et al. Brain imaging changes associated with risk factors for cardiovascular and cerebrovascular disease in asymptomatic patients. JACC Cardiovasc. Imaging 7, 1039-1053 (2014).

16. Fuhrmann, D. et al. Strong and specific associations between cardiovascular risk factors and white matter micro- and macrostructure in healthy aging. Neurobiol. Aging 74, 46-55 (2019).

17. Wardlaw, J. M. et al. Neuroimaging standards for research into small vessel disease and its contribution to ageing and neurodegeneration. Lancet Neurol. 12, 822-838 (2013).

18. Debette, S. \& Markus, H. S. The clinical importance of white matter hyperintensities on brain magnetic resonance imaging: systematic review and meta-analysis. BMJ Clin. Res. Ed. 341, 3666 (2010).

19. Gilbert, S. J. \& Burgess, P. W. Executive function. Curr. Biol. 18, R110-R114 (2008).

20. Poulin, V., Korner-Bitensky, N., Dawson, D. R. \& Bherer, L. Efficacy of executive function interventions after stroke: a systematic review. Top. Stroke Rehabil. 19, 158-171 (2012).

21. Montembeault, M. et al. The impact of aging on gray matter structural covariance networks. NeuroImage 63, 754-759 (2012).

22. Smith, S. M. et al. Correspondence of the brain's functional architecture during activation and rest. Proc. Natl. Acad. Sci. U.S.A. 106, 13040-13045 (2009).

23. Parlatini, V. et al. Functional segregation and integration within fronto-parietal networks. Neuroimage 146, 367-375 (2017).

24. Ptak, R. The frontoparietal attention network of the human brain: action, saliency, and a priority map of the environment. Neurosci. Rev. J. Bringing Neurobiol. Neurol. Psychiatry 18, 502-515 (2012).

25. Wardlaw, J. M., Valdés Hernández, M. C. \& Muñoz-Maniega, S. What are White Matter Hyperintensities Made of? J Am Heart Assoc 4, (2015).

26. Salat, D. H. et al. Inter-individual variation in blood pressure is associated with regional white matter integrity in generally healthy older adults. NeuroImage 59, 181-192 (2012).

27. Li, X. et al. Disrupted frontoparietal network mediates white matter structure dysfunction associated with cognitive decline in hypertension patients. J. Neurosci. 35, 10015-10024 (2015).

28. O'Sullivan, M. et al. Diffusion tensor MRI correlates with executive dysfunction in patients with ischaemic leukoaraiosis. J. Neurol. Neurosurg. Psychiatry 75, 441-447 (2004).

29. Brodtmann, A. et al. Charting cognitive and volumetric trajectories after stroke: protocol for the Cognition and Neocortical Volume After Stroke (CANVAS) study. Int. J. Stroke Off. J. Int. Stroke Soc. 9, 824-828 (2014).

30. Tombaugh, T. N. Trail making test A and B: normative data stratified by age and education. Arch. Clin. Neuropsychol. 19, 203-214 (2004).

31. Sunderland, T. et al. Clock drawing in Alzheimer's disease. J. Am. Geriatr. Soc. 37, 725-729 (1989). 
32. Fastenau, P. S., Denburg, N. L. \& Hufford, B. J. Adult norms for the rey-osterrieth complex figure test and for supplemental recognition and matching trials from the extended complex figure test. Clin. Neuropsychol. 13, 30-47 (1999).

33. Lloyd-Jones, D. M. et al. Framingham risk score and prediction of lifetime risk for coronary heart disease. Am. J. Cardiol. 94, 20-24 (2004).

34. Ithapu, V. et al. Extracting and summarizing white matter hyperintensities using supervised segmentation methods in Alzheimer's disease risk and aging studies. Hum. Brain Mapp. 35, 4219-4235 (2014).

35. Hua, K. et al. Tract probability maps in stereotaxic spaces: analyses of white matter anatomy and tract-specific quantification. Neuroimage 39, 336-347 (2008).

36. Rosseel, Y. lavaan: an R package for structural equation modeling. J. Stat. Softw. 48, 1-36 (2012).

37. Bamford, J., Sandercock, P., Dennis, M., Warlow, C. \& Burn, J. Classification and natural history of clinically identifiable subtypes of cerebral infarction. The Lancet 337, 1521-1526 (1991).

38. Hooper, D., Coughlan, J. \& Mullen, M. R. Structural equation modelling: guidelines for determining model fit. Electron. J. Bus. Res. Methods 6, 53-59 (2008).

39. Kloppenborg, R., Nederkoorn, P., Geerlings, M. \& van den Berg, E. Presence and progression of white matter hyperintensities and cognition: a meta-analysis. Neurology 82, 2127-2138 (2014).

40. A lateralized brain network for visuospatial attention | Nature Neuroscience. https://www.nature.com/articles/nn.2905.

\section{Acknowledgements}

We would like to thank our patients and their carers for their time taking part in the experiment over a number of years. This work was supported by the National Health and Medical Research Council project grant number APP1020526, the Brain Foundation, Wicking Trust, Collie Trust, and Sidney and Fiona Myer Family Foundation.

\section{Author contributions}

Data collection E.W., M.S.K., Data preprocessing and preparation E.W., M.S.K. and N.E. Study design, analysis and manuscript preparation M.V. Discussion, interpretation and manuscript editing A.B., E.W., M.S.K., N.E., M.V.

\section{Competing interests}

The authors declare no competing interests.

\section{Additional information}

Correspondence and requests for materials should be addressed to M.V.

Reprints and permissions information is available at www.nature.com/reprints.

Publisher's note Springer Nature remains neutral with regard to jurisdictional claims in published maps and institutional affiliations.

(c) (i) Open Access This article is licensed under a Creative Commons Attribution 4.0 International (c) License, which permits use, sharing, adaptation, distribution and reproduction in any medium or format, as long as you give appropriate credit to the original author(s) and the source, provide a link to the Creative Commons licence, and indicate if changes were made. The images or other third party material in this article are included in the article's Creative Commons licence, unless indicated otherwise in a credit line to the material. If material is not included in the article's Creative Commons licence and your intended use is not permitted by statutory regulation or exceeds the permitted use, you will need to obtain permission directly from the copyright holder. To view a copy of this licence, visit http://creativecommons.org/licenses/by/4.0/.

(C) The Author(s) 2020 